\title{
Successful Treatment of Alopecia Areata Barbae with Platelet-rich Plasma
}

\author{
Hanno Pototschnig ${ }^{1}$, Maximilian T. Madl ${ }^{2}$ \\ 1. Regenerative Medicine, Munich Medical Esthetic, Munich, DEU 2. Orthopaedics, Privatpraxis Josephsburg, Munich, \\ DEU
}

Corresponding author: Hanno Pototschnig, hanno.pototschnig@gmx.at

\begin{abstract}
Alopecia areata (AA) is a frequent autoimmune disorder in which inflammatory cells attack the hair follicles. AA affecting the beard area is well known and is referred to as alopecia areata barbae (AAB) when involvement is limited exclusively to the beard. Currently, no guidelines are established for specific therapeutic approaches for this condition. We present a case of a healthy 30 -year-old male suffering from $\mathrm{AAB}$. Three injections of platelet-rich plasma (PRP) with six-week intervals were applied. Stabilization of the condition was noted at the first follow-up (before the second injection), initial minimal hair regrowth was noted at the second follow-up (before the third injection) and robust regrowth at the one-year followup. To our knowledge, this case is the first report of a successful treatment of AAB using PRP. PRP represents a new, safe and potentially effective treatment option for AAB. More studies will be necessary to determine the efficacy of this treatment compared to conventional therapy.
\end{abstract}

Categories: Dermatology, Plastic Surgery, Miscellaneous

Keywords: aesthetics, acp, alopecia areata, alopecia areata barbae, autologous conditioned plasma, beard, dermatology, hair loss, platelet-rich plasma, trichology

\section{Introduction}

With a lifetime prevalence of approximately $2 \%$, alopecia areata (AA) is a frequent autoimmune disorder in which inflammatory cells attack the hair follicles [1]. Several subtypes of AA exist. AA affecting the beard area is well known and is referred to as AA of the beard (BAA) or AA barbae (AAB) when involvement is limited exclusively to the beard. Frequently steroid injection is used as a treatment modality, but currently, no guidelines are established for specific therapeutic approaches for BAA or AAB $[2,3]$. Furthermore, no randomized controlled trials for the treatment of BAA have been undertaken [4].

\section{Case Presentation}

We present a case of a patient with $\mathrm{AAB}$ who had robust regrowth with platelet-rich plasma (PRP) injections. A healthy 30-year-old male with AAB presented with the wish of PRP treatment for aesthetic improvement in the area of his beard. Progressing patchy AAB was present for over two years. Scalp, eyelashes, eyebrows and nails were unaffected. Apart from the AAB, the patient did not suffer from any diseases and did not take any medication. Treatment was administered three times at six-week intervals with autologous PRP. PRP has been prepared as shown in Video 1.

\section{VIDEO 1: Preparation of PRP.}

PRP, platelet-rich plasma.

\section{View video here: https://vimeo.com/383748386}

For each treatment, $30 \mathrm{ml}$ of blood was taken from the patient via a 21-gauge scalp vein set into two ACP double syringes (Arthrex Inc, Naples, FL). Directly after this, the syringes were centrifuged horizontally for five minutes at approximately $350 \mathrm{G}$. In each syringe, the PRP settled in the upper third of the syringe (approximately $5 \mathrm{ml}$ ), and was drawn into the inner syringe. The inner syringe was twisted out. Following topical anesthesia and disinfection, PRP injections were performed $1 \mathrm{~cm}$ apart at a depth of 2-3 mm in serial puncture technique in the affected area (approximately $80 \mathrm{~cm}^{2}$ ) using 34 gauge needles. Additional methods of platelet activation e.g. addition of thrombin or calcium gluconate were not used in the treatment protocol for this patient. Overall, the procedure was well tolerated. The typical burning sensation during injection was not reported. This could be explained due to the fact that the PRP contained no anticoagulants, which have an unfavorable $\mathrm{pH}$ value and could therefore cause a burning sensation. Apart from minimal discomfort within the first 36 hours after injection, no adverse effects were reported. Stabilization of the condition was noted at the first follow-up (before the second injection), minimal hair regrowth was noted at the second follow-up (before the third injection) and robust regrowth at the one-year follow-up (Figure 1). 


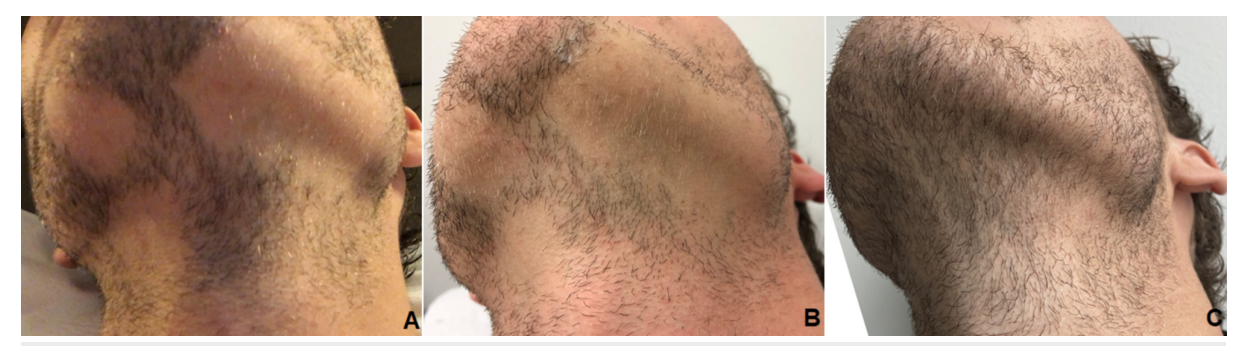

FIGURE 1: Initial status (A), minimal regrowth six weeks after the
second injection (B), robust regrowth at one-year follow-up (C).

\section{Discussion}

PRP is considered to initiate wound healing through secretion of various growth factors and cytokines. Antiapoptotic effects on dermal papilla cells and prolongation of the anagen phase through increases in Bcatenin and fibroblast growth factor-7 have been shown [5]. Over the last years, PRP became more and more popular for the treatment of hairloss. Especially for androgenetic alopecia a wide body of evidence has recently emerged, demonstrating a positive response from PRP treatments. According to the review from Chen et al., 21 studies reported positive outcomes by objective criteria (88\%), while three suggested that there was no clinical improvement, although in two of these studies patients still reported increased satisfaction. There were no complications reported other than transient edema/erythema and pain/headache associated with the procedure. PRP is considered a low-risk intervention to treat androgentic alopecia associated with good patient satisfaction and objective improvements in outcomes [6].

In comparison to androgenetic alopecia, fewer studies have investigated the efficacy of PRP for AA. Donovan reported successful treatment of corticosteroid-resistant ophiasis-type AA with PRP [7]. A recent trial showed that patients treated with topical minoxidil and PRP have both significantly increased hair regrowth compared to placebo and patients treated with PRP have a significantly earlier response than topical minoxidil [8]. In a randomized study, PRP demonstrated significantly improved hair regrowth compared to placebo and triamcinolone scalp injections without any noted adverse events [9]. However, a variable effect was reported in another trial in chronic severe AA [10].

To our knowledge, our case is the first report of a successful treatment of AAB using PRP. PRP represents a new, safe and potentially effective treatment option for $A A B$. More studies will be necessary to determine the efficacy of this treatment compared to conventional therapy.

\section{Conclusions}

An emerging body of evidence suggests that PRP is a safe and effective treatment modality for androgenetic alopecia. Initial studies report promising results after PRP injections for AA as well. Concerning the subtype of $A A B$, our case seems to be the first report of a successful treatment. Further studies are needed to confirm these encouraging findings.

\section{Additional Information \\ Disclosures}

Human subjects: Consent was obtained by all participants in this study. Conflicts of interest: In compliance with the ICMJE uniform disclosure form, all authors declare the following: Payment/services info: All authors have declared that no financial support was received from any organization for the submitted work. Financial relationships: Hanno Pototschnig, Maximilian T. Madl declare(s) employment from Arthrex GmbH. Other relationships: All authors have declared that there are no other relationships or activities that could appear to have influenced the submitted work.

\section{References}

1. Villasante Fricke AC, Miteva M: Epidemiology and burden of alopecia areata: a systematic review . Clin Cosmet Investig Dermatol. 2015, 8:397-403. 10.2147/CCID.S53985

2. Messenger AG, McKillop J, Farrant P, McDonagh AJ, Sladden M: British Association of Dermatologists' guidelines for the management of alopecia areata 2012. Br J Dermatol. 2012, 166:916-926. 10.1111/j.13652133.2012.10955.x

3. Alkhalifah A, Alsantali A, Wang E, McElwee KJ, Shapiro J: Alopecia areata update: part II. Treatment. J Am Acad Dermatol. 2010, 62:191-202. 10.1016/j.jaad.2009.10.031

4. Cervantes J, Fertig RM, Maddy A, Tosti A: Alopecia areata of the beard: a review of the literature . Am J Clin Dermatol. 2017, 18:789-796. 10.1007/s40257-017-0297-6

5. Li ZJ, Choi HI, Choi DK, et al.: Autologous platelet-rich plasma: a potential therapeutic tool for promoting hair growth. Dermatol Surg. 2012, 38:1040-1046. 10.1111/j.1524-4725.2012.02394.x

6. Chen JX, Justicz N, Lee LN: Platelet-rich plasma for the treatment of androgenic alopecia: a systematic review. Facial Plast Surg. 2018, 34:631-640. 10.1055/s-0038-1660845 


\section{Cureus}

7. Donovan J: Successful treatment of corticosteroid-resistant ophiasis-type alopecia areata (AA) with platelet-rich plasma (PRP). JAAD Case Rep. 2015, 25:305-307. 10.1016/j.jdcr.2015.07.004

8. El Taieb MA, Ibrahim H, Nada EA, Seif Al-Din M: Platelets rich plasma versus minoxidil 5\% in treatment of alopecia areata: a trichoscopic evaluation. Dermatol Ther. 2017, 30:e12437. 10.1111/dth.12437

9. Trink A, Sorbellini E, Bezzola P, et al.: A randomized, double-blind, placebo- and active-controlled, halfhead study to evaluate the effects of platelet-rich plasma on alopecia areata. Br J Dermatol. 2013, 169:690694. 10.1111/bjd.12397

10. d'Ovidio R, Roberto M: Limited effectiveness of platelet-rich-plasma treatment on chronic severe alopecia areata. Hair Ther Transplant. 2014, 4:116. 10.4172/2167-0951.1000116 